\title{
Global Practical Output Tracking of Inherently Nonlinear Systems Using Continuously Differentiable Controllers
}

\author{
Keylan Alimhan, ${ }^{1,2}$ Naohisa Otsuka, ${ }^{2}$ \\ Abilmazhin A. Adamov, ${ }^{1}$ and Maksat N. Kalimoldayev ${ }^{3}$ \\ ${ }^{1}$ L.N. Gumilyov Eurasian National University, Astana, Kazakhstan \\ ${ }^{2}$ School of Science and Engineering, Tokyo Denki University, Saitama 350-0394, Japan \\ ${ }^{3}$ Institute of Information and Computing Technologies, Almaty, Kazakhstan \\ Correspondence should be addressed to Naohisa Otsuka; otsuka@mail.dendai.ac.jp
}

Received 16 March 2015; Revised 17 May 2015; Accepted 20 May 2015

Academic Editor: Yan-Wu Wang

Copyright (C) 2015 Keylan Alimhan et al. This is an open access article distributed under the Creative Commons Attribution License, which permits unrestricted use, distribution, and reproduction in any medium, provided the original work is properly cited.

This paper considers the global practical output tracking problem by at least continuously differentiable $\left(C^{1}\right)$ state feedback for a class of uncertain nonlinear systems whose linearization around the origin may contain uncontrollable modes. Based on utilizing the homogeneous domination approach, we not only propose conditions of constructing a global continuously differentiable $\left(C^{1}\right)$ controller, but also provide explicit design schemes for such systems. Finally, a numerical example demonstrates the effectiveness of the result.

\section{Introduction}

The problem of global output tracking control of nonlinear systems is one of the most important and challenging problems in the field of nonlinear control. One of recent focuses in the nonlinear control research is the global practical output tracking problem for a class of inherently nonlinear systems, described by the following equations:

$$
\begin{aligned}
& \dot{z}_{i}=z_{i+1}^{p_{i}}+f_{i}(t, z, u), \quad i=1, \ldots, n-1, \\
& \dot{z}_{n}=u^{p_{n}}+f_{n}(t, z, u), \\
& y=z_{1},
\end{aligned}
$$

where $z=\left(z_{1}, \ldots, z_{n}\right)^{\mathrm{T}} \in R^{n}$ and $u \in R$ are the system state and the control input, respectively. For $i=1, \ldots, n, f_{i}(t, z, u)$ are unknown continuous $\left(C^{0}\right)$ nonlinear functions of the states and the control input and the power $p_{i} \in R_{\text {odd }}^{+}(i=$ $1, \ldots, n-1)$ is a positive odd integer or a positive ratio of odd integers with $p_{n}:=1$.

The uncertain system (1) represents a general class of nonlinear systems considered in the nonlinear control literature. When $p_{i}=1, i=1, \ldots, n$, system (1) reduces to the well-known feedback linearizable form, for which numerous design methodologies are developed; see [1-5] and the references therein. For the case that any one of the powers $p_{i}(i=1,2, \ldots, n)$ is greater than 1 , system (1) is known as the power integrator system whose Jacobian linearization is uncontrollable. In recent years, the problem of global practical output tracking control of the power integrator systems in form (1) has been studied extensively with various restrictions on the integrator powers and the additive functions $f_{i}(t, z, u)$ 's, which directly influence the availability of smooth or nonsmooth controllers; see [6-10] and the references therein. For details, in [6], practical output tracking via state feedback for high-order $\left(p_{i} \geq 1, i=\right.$ $1, \ldots, n)$ nonlinear systems was considered. Further, in $[8$, 9], the practical output feedback tracking problem was also investigated for a class of nonlinear systems with higherorder growing unmeasurable states, extending the results on stabilization in [11, 12].

For the more general nonlinear systems with arbitrary $p_{i}(>0)$ 's, existing results toward the global output tracking problem for system (1) can be found in the literature. The global stabilization problem of system (1) for $p_{i}>0$ (not restricted to be larger than or equal to one) has been 
studied for nonlinear systems in $[13,14]$. In [13], a continuous controller under a certain nonlinear growth condition is studied.

The techniques from $[11,14]$ were recently extended in $[10,15]$ to the practical output tracking problem for nonlinear systems (1) by a continuous state feedback controller. However, from the practical point of view, the smoothness of the controllers is always desired because controllers at least $C^{1}$ avoid the infinity controller gains around the origin and guarantee the uniqueness of the solution $[16,17]$. Initial efforts were made in [18] to obtain $C^{1}$ or smooth controllers by upgrading the unified homogeneous degree to a set of decreasing homogeneous degrees to solve the global stabilization problem of system (1) for $p_{i}>0$. It was shown that these monotone degrees gave us much flexibility in the controller design, which will lead to some nicer features for the controlled system.

In this paper, we will further generalize the results in [18] to solve the practical output tracking problem. This work will develop a detailed recursive design method which constructs a series of integral Lyapunov functions as well as the explicit formula of the continuously differentiable controllers.

Throughout this study we use the following notations.

Notations. $R^{+}$denotes the set of all the nonnegative real numbers and $R^{n}$ denotes the real $n$-dimensional space. A function $f: R^{n} \rightarrow R$ is said to be $C^{k}$-function, if its partial derivatives exist and are continuous up to order $k, 1 \leq k<$ $\infty$. A $C^{0}$ function means it is continuous. A $C^{\infty}$ function means it is smooth; that is, it has continuous partial derivatives of any order. The arguments of functions (or functionals) are sometimes omitted or simplified; for instance, we sometimes denote a function $f(x(t))$ by $f(x), f(\cdot)$, or $f$.

\section{Problem Statement and Preliminaries}

The purpose of this paper is to solve the problem of global practical output tracking by state feedback. Let $y_{r}(t)$ be a time-varying $C^{1}$-bounded on $t \in[0,+\infty)$ reference signal and, for any given tolerance $\varepsilon>0$, design a continuously differentiable state feedback controller of the form

$$
u=u\left(z, y_{r}(t)\right)
$$

such that

(i) all the states of the closed-loop system (1)-(2) are welldefined on $t \in[0,+\infty)$ and globally bounded;

(ii) for any initial condition $z(0) \in R^{n}$ there is a finite time $T>0$, such that

$$
\left|y(t)-y_{r}(t)\right|=\left|z_{1}(t)-y_{r}(t)\right|<\varepsilon, \quad \forall t \geq T>0 .
$$

To construct a global practical output tracking $C^{1}$ controller for nonlinear system (1), we introduce the following assumptions.

Assumption 1. For $i=1, \ldots, n$, there are decreasing constants $\tau_{i}=q_{i} / d_{i}$, with an even integer $q_{i}$ and an odd integer $d_{i}\left(\tau_{1} \geq\right.$ $\tau_{2} \geq \cdots \geq \tau_{n} \geq 0$ ), such that (i) the following holds:

$$
\begin{aligned}
& \left|f_{i}\left(x_{1}, \ldots, x_{i}\right)\right| \\
& \quad \leq b_{i}\left(x_{1}, \ldots, x_{i}\right)\left(\left|x_{1}\right|^{\left(r_{i}+\tau_{i}\right) / r_{1}}+\cdots+\left|x_{i}\right|^{\left(r_{i}+\tau_{i}\right) / r_{i}}\right),
\end{aligned}
$$

where $b_{i}\left(x_{1}, \ldots, x_{i}\right)>0$ are smooth functions and $r_{i}$ 's are defined as

$$
\begin{aligned}
r_{1} & =1, \\
r_{i+1} p_{i} & =r_{i}+\tau_{i}, \quad i=1, \ldots, n ;
\end{aligned}
$$

(ii) the $r_{i}$ 's defined by (i) satisfy the following condition:

$$
r_{n}+\tau_{n} \geq \max _{1 \leq i \leq n}\left\{r_{i}\right\}
$$

Assumption 2. The reference signal $y_{r}(t)$ is continuously differentiable. Moreover, there is a known constant $M>0$, such that

$$
\left|y_{r}(t)\right|+\left|\dot{y}_{r}(t)\right| \leq M, \quad \forall t \in[0, \infty) .
$$

This section cites some definitions and technical lemmas which are used in the main body of this investigation.

Next, we will present several useful lemmas borrowed from $[4,13,14,19]$, which will play an important role in our later controller design.

Lemma 3. For all $x, y \in R$ and a constant $p \geq 1$, the following inequalities hold:

(i) $|x+y|^{p} \leq 2^{p-1}\left|x^{p}+y^{p}\right|,(|x|+|y|)^{1 / p} \leq|x|^{1 / p}+|y|^{1 / p} \leq$ $2^{(p-1) / p}(|x|+|y|)^{1 / p}$

if $p \in R_{\text {odd }}^{\geq 1}$ then

(ii) $|x-y|^{p} \leq 2^{p-1}\left|x^{p}-y^{p}\right|,\left|x^{1 / p}-y^{1 / p}\right| \leq 2^{(p-1) / p} \mid x-$ $\left.y\right|^{1 / p}$.

Lemma 4. For given positive real numbers $m, n$ and a positive function $a(x, y)$, there exists a positive function $c(x, y)$, such that

$$
a(x, y)|x|^{m}|y|^{n} \leq c(x, y)|x|^{m+n}
$$

$$
\begin{aligned}
& +\frac{n}{n+m}\left(\frac{m}{(m+n) c(x, y)}\right)^{m / n} \\
& \cdot a(x, y)^{(m+n) / n}|y|^{m+n} .
\end{aligned}
$$

Lemma 5. For any positive real numbers $x, y$ and $m \geq 1$, the following inequality holds:

$$
x \leq y+\left(\frac{x}{m}\right)^{m}\left(\frac{(m-1)}{y}\right)^{m-1} .
$$

Lemma 6. Let $x_{1}, \ldots, x_{n}, p>0$ be real numbers. Then, the following inequality holds:

$$
\left(x_{1}+\cdots+x_{n}\right)^{p} \leq \max \left(n^{p-1}, 1\right) \cdot\left(x_{1}^{p}+\cdots+x_{n}^{p}\right) .
$$




\section{Continuously Differentiable State Feedback Controller Design}

In this section, we will construct a continuously differentiable state feedback tracking controller which is addressed in a step-by-step manner for system (1).

Theorem 7. Under Assumptions 1-2, the global practical output tracking problem of system (1) can be solved by a continuously differentiable $\left(C^{1}\right)$ state feedback controller of form (2).

Proof. Let $\rho \in R_{\text {odd }}^{+}$be a constant satisfying $\rho \geq \max _{1 \leq i \leq n}\left\{r_{i}+\right.$ $\left.\tau_{i}\right\}$, where $\tau_{i}$ and $r_{i}$ are defined by Assumption 1 .

Let $x_{1}=z_{1}-y_{r}$ and given $x_{i}=z_{i}, i=2, \ldots, n$. Then we have

$$
\begin{aligned}
& \dot{x}_{1}=x_{2}^{p_{1}}+f_{1}\left(x_{1}+y_{r}\right)-\dot{y}_{r}(t), \\
& \dot{x}_{j}=x_{j+1}^{p_{j}}+f_{j}\left(x_{1}+y_{r}, x_{2}, \ldots, x_{j}\right), \\
& \quad j=2, \ldots, n-1, \\
& \dot{x}_{n}=u+f_{n-1}\left(x_{1}+y_{r}, x_{2}, \ldots, x_{n-1}, x_{n}\right), \\
& y=x_{1}+y_{r} .
\end{aligned}
$$

Initial Step. Choose

$$
\begin{aligned}
V_{1}\left(x_{1}\right) & =\int_{0}^{x_{1}}\left(s^{\left(r_{n}+\tau_{n}\right) / r_{1}}-0\right)^{\left(2 \rho-r_{1}-\tau_{1}\right) /\left(r_{n}+\tau_{n}\right)} d s \\
& =\frac{r_{1}}{2 \rho-\tau_{1}} x_{1}^{\left(2 \rho-\tau_{1}\right) / r_{1}}
\end{aligned}
$$

which is positive definite, proper, and $C^{1}$ due to the fact that $2 \rho-\tau_{1} \geq 2 r_{1}+\tau_{1}$. Then, the time derivative of $V_{1}\left(x_{1}\right)$ along the trajectory of (1) is

$$
\dot{V}_{1}\left(x_{1}\right)=x_{1}^{\left(2 \rho-\tau_{1}-r_{1}\right) / r_{1}}\left[x_{2}^{p_{1}}+f_{1}\left(x_{1}+y_{r}\right)-\dot{y}_{r}\right] .
$$

Further, it follows from Assumptions 1(ii) and 2 and Lemmas 3-5 that

$$
\begin{aligned}
\dot{V}_{1}\left(x_{1}\right) \leq & x_{1}^{\left(2 \rho-\tau_{1}-r_{1}\right) / r_{1}} x_{2}^{* p_{1}} \\
& +x_{1}^{\left(2 \rho-\tau_{1}-r_{1}\right) / r_{1}}\left(x_{2}^{p_{1}}-x_{2}^{* p_{1}}\right) \\
& +x_{1}^{\left(2 \rho-\tau_{1}-r_{1}\right) / r_{1}} b_{1}\left(x_{1}+y_{r}\right)\left|x_{1}+y_{r}\right|^{\left(\tau_{1}+r_{1}\right) / r_{1}} \\
& +M x_{1}^{\left(2 \rho-\tau_{1}-r_{1}\right) / r_{1}} \\
\leq & x_{1}^{\left(2 \rho-\tau_{1}-r_{1}\right) / r_{1}} x_{2}^{* p_{1}} \\
& +x_{1}^{\left(2 \rho-\tau_{1}-r_{1}\right) / r_{1}}\left(x_{2}^{p_{1}}-x_{2}^{* p_{1}}\right) \\
& +2^{\tau_{1} / r_{1}} x_{1}^{2 \rho / r_{1}} \tilde{b}_{1}\left(x_{1}\right)+B_{1}\left(x_{1}\right) x_{1}^{\left(2 \rho-\tau_{1}-r_{1}\right) / r_{1}}
\end{aligned}
$$

$$
\begin{aligned}
\leq & x_{1}^{\left(2 \rho-\tau_{1}-r_{1}\right) / r_{1}} x_{2}^{* p_{1}} \\
& +x_{1}^{\left(2 \rho-\tau_{1}-r_{1}\right) / r_{1}}\left(x_{2}^{p_{1}}-x_{2}^{* p_{1}}\right) \\
& +\left(2^{\tau_{1} / r_{1}} \widetilde{b}_{1}\left(x_{1}\right)+\widetilde{B}_{1}\left(x_{1}\right)\right) x_{1}^{2 \rho / r_{1}}+\delta,
\end{aligned}
$$

where $\widetilde{B}_{1}\left(x_{1}\right)=\left[B_{1}\left(x_{1}\right)\right]^{2 \rho /\left(2 \rho-\tau_{1}-r_{1}\right)} / \delta^{\left(\tau_{1}+r_{1}\right) /\left(2 \rho-\tau_{1}-r_{1}\right)}$, $B_{1}\left(x_{1}\right) \geq\left(2^{\tau_{1} / r_{1}} b_{1}\left(x_{1}+y_{r}\right) M^{\left(\tau_{1}+r_{1}\right) / r_{1}}+M\right)$, and $\delta>0$ is any real constant.

Since $b_{1}(\cdot)$ is smooth function and $y_{r}(t)$ is bounded, we can choose $\tilde{b}_{1}\left(x_{1}\right) \geq b_{1}\left(x_{1}+y_{r}\right)$.

Design the virtual controller $x_{2}^{* p_{1}}$ as

$$
\begin{aligned}
x_{2}^{* p_{1}} & =-x_{1}^{\left(\tau_{1}+r_{1}\right) / r_{1}} \beta_{1}\left(x_{1}\right) \\
& =-\left(x_{1}^{\left(\tau_{n}+r_{n}\right) / r_{1}}\right)^{\left(\tau_{1}+r_{1}\right) /\left(\tau_{n}+r_{n}\right)} \beta_{1}\left(x_{1}\right) \\
& =-\xi_{1}^{r_{2} p_{1} /\left(\tau_{n}+r_{n}\right)} \beta_{1}\left(x_{1}\right)
\end{aligned}
$$

with a smooth function $\beta_{1}\left(x_{1}\right)=n+2^{\tau_{1} / r_{1}} \widetilde{b}_{1}\left(x_{1}\right)+\widetilde{B}_{1}\left(x_{1}\right)$, and then we have

$$
\dot{V}_{1}\left(x_{1}\right) \leq-n x_{1}^{2 \rho / r_{1}}+x_{1}^{\left(2 \rho-\tau_{1}-r_{1}\right) / r_{1}}\left(x_{2}^{p_{1}}-x_{2}^{* p_{1}}\right)+\delta .
$$

Inductive Step. Suppose, at step $(k-1)$, there exist a series of smooth functions $\beta_{i}\left(x_{1}, \ldots, x_{i}\right)>0, i=1, \ldots, k-1$, with the following virtual controllers:

$$
\begin{aligned}
& x_{1}^{*}=0 \text {, } \\
& x_{2}^{* p_{1}}=-\xi_{1}^{r_{2} p_{1} /\left(r_{n}+\tau_{n}\right)} \beta_{1}\left(x_{1}\right) \text {, } \\
& x_{k}^{* p_{k-1}}=-\xi_{k-1}^{r_{k} p_{k-1} /\left(r_{n}+\tau_{n}\right)} \beta_{k-1}\left(x_{1}, \ldots, x_{k-1}\right), \\
& \xi_{1}=x_{1}^{\left(r_{n}+\tau_{n}\right) / r_{1}}-x_{1}^{*\left(r_{n}+\tau_{n}\right) / r_{1}} \\
& \xi_{2}=x_{2}^{\left(r_{n}+\tau_{n}\right) / r_{2}}-x_{2}^{*\left(r_{n}+\tau_{n}\right) / r_{2}} \\
& \xi_{k}=x_{k}^{\left(r_{n}+\tau_{n}\right) / r_{k}}-x_{k}^{*\left(r_{n}+\tau_{n}\right) / r_{k}},
\end{aligned}
$$

such that

$$
\begin{aligned}
\dot{V}_{k-1} & \left(x_{1}, \ldots, x_{k-1}\right) \\
\leq & -(n-k+2)\left(\xi_{1}^{2 \rho /\left(r_{n}+\tau_{n}\right)}+\cdots+\xi_{k-1}^{2 \rho /\left(r_{n}+\tau_{n}\right)}\right) \\
& +\xi_{k-1}^{\left(2 \rho-r_{k-1}-\tau_{k-1}\right) /\left(r_{n}+\tau_{n}\right)}\left(x_{k}^{p_{k-1}}-x_{k}^{* p_{k-1}}\right) \\
& +(k-1) \delta .
\end{aligned}
$$


We claim that (18) also holds at step $k$. To prove this claim, consider the Lyapunov function

$$
\begin{aligned}
V_{k} & \left(x_{1}, \ldots, x_{k}\right) \\
= & V_{k-1}\left(x_{1}, \ldots, x_{k-1}\right)+W_{k}\left(x_{1}, \ldots, x_{k}\right) \\
= & V_{k-1}\left(x_{1}, \ldots, x_{k-1}\right) \\
\quad & \quad \int_{x_{k}^{*}}^{x_{k}}\left(s^{\left(r_{n}+\tau_{n}\right) / r_{k}}-x_{k}^{*\left(r_{n}+\tau_{n}\right) / r_{k}}\right)^{\left(2 \rho-r_{k}-\tau_{k}\right) /\left(r_{n}+\tau_{n}\right)} d s .
\end{aligned}
$$

The function $V_{k}\left(x_{1}, x_{2}, \ldots, x_{k}\right)$ can be shown to be $C^{1}$, proper, and positive definite with the following property: for $i=$ $1, \ldots, k-1$,

$$
\begin{aligned}
& \frac{\partial W_{k}}{\partial x_{i}}=-\frac{2 \rho-r_{k}-\tau_{k}}{\left(r_{n}+\tau_{n}\right)} \\
& \quad \cdot \int_{x_{k}^{*}}^{x_{k}}\left(s^{\left(r_{n}+\tau_{n}\right) / r_{k}}-x_{k}^{*\left(r_{n}+\tau_{n}\right) / r_{k}}\right)^{\left(2 \rho-r_{k}-\tau_{k}\right) /\left(r_{n}+\tau_{n}\right)-1} d s \\
& \quad \cdot \frac{\partial\left(x_{k}^{*\left(r_{n}+\tau_{n}\right) / r_{k}}\right)}{\partial x_{i}}, \\
& \frac{\partial W_{k}}{\partial x_{k}}=\left(x^{\left(r_{n}+\tau_{n}\right) / r_{k}}-x_{k}^{*\left(r_{n}+\tau_{n}\right) / r_{k}}\right)^{\left(2 \rho-r_{k}-\tau_{k}\right) /\left(r_{n}+\tau_{n}\right)} \\
& \quad=\xi_{k}^{\left(2 \rho-r_{k}-\tau_{k}\right) /\left(r_{n}+\tau_{n}\right)}
\end{aligned}
$$

and there is a known constant $L>0$ such that

$$
W_{k} \geq L\left(x_{k}-x_{k}^{*}\right)^{\left(2 \rho-r_{k}-\tau_{k}\right) / r_{k}}
$$

Proofs of these properties proceed just in the same way as in the proofs for [20, Propositions 1 and 2] and [21], where the set of positive odd integers is considered instead of $R_{\text {odd }}$ which is used in this paper.

With these properties, we obtain

$$
\begin{aligned}
& \dot{V}_{k}\left(x_{1}, \ldots, x_{k}\right) \leq-(n-k+2)\left(\xi_{1}^{2 \rho /\left(r_{n}+\tau_{n}\right)}+\cdots\right. \\
& \left.\quad+\xi_{k-1}^{2 \rho /\left(r_{n}+\tau_{n}\right)}\right)+\xi_{k-1}^{\left(2 \rho-r_{k-1}-\tau_{k-1}\right) /\left(r_{n}+\tau_{n}\right)}\left(x_{k}^{p_{k-1}}-x_{k}^{* p_{k-1}}\right) \\
& \quad+(k-1) \delta+\xi_{k}^{\left(2 \rho-r_{k}-\tau_{k}\right) /\left(r_{n}+\tau_{n}\right)}\left(x_{k+1}^{* p_{k}}\right. \\
& \left.\quad+f_{k}\left(x_{1}+y_{r}, x_{2}, \ldots, x_{k}\right)\right)+\sum_{i=1}^{k-1} \frac{\partial W_{k}}{\partial x_{i}} \dot{x}_{i} \\
& \quad+\xi_{k}^{\left(2 \rho-r_{k}-\tau_{k}\right) /\left(r_{n}+\tau_{n}\right)}\left(x_{k+1}^{p_{k}}-x_{k+1}^{* p_{k}}\right)
\end{aligned}
$$

for a virtual controller $x_{k+1}^{* p_{k}}$ to be determined later. In order to proceed further, a bounding estimate for each term in the right hand side of (22) is needed. The terms in (22) can be estimated using Propositions A.1-A.3 in the appendix.
Substituting the results of Propositions A.1-A.3 into (22), we arrive at

$$
\begin{aligned}
\dot{V}_{k}( & \left.x_{1}, \ldots, x_{k}\right) \\
\leq & -(n-k+1)\left(\xi_{1}^{2 \rho /\left(r_{n}+\tau_{n}\right)}+\cdots+\xi_{k-1}^{2 \rho /\left(r_{n}+\tau_{n}\right)}\right) \\
& +\xi_{k}^{\left(2 \rho-r_{k}-\tau_{k}\right) /\left(r_{n}+\tau_{n}\right)} x_{k+1}^{* p_{k}} \\
& +\widetilde{\kappa}_{k}\left(x_{1}, \ldots, x_{k}\right) \xi_{k}^{2 \rho /\left(r_{n}+\tau_{n}\right)} \\
& +\xi_{k}^{\left(2 \rho-r_{k}-\tau_{k}\right) /\left(r_{n}+\tau_{n}\right)}\left(x_{k+1}^{p_{k}}-x_{k+1}^{* p_{k}}\right)+k \delta,
\end{aligned}
$$

where

$$
\begin{aligned}
& \widetilde{\kappa}_{k}\left(x_{1}, \ldots, x_{k}\right)\left(\geq g_{k}\left(x_{1}, \ldots, x_{k}\right)+h_{k}\left(x_{1}, \ldots, x_{k}\right)\right. \\
& \left.\quad+l_{k}\left(x_{1}, \ldots, x_{k}\right)\right)
\end{aligned}
$$

is a smooth positive function.

Therefore, if we take the virtual control $x_{k+1}^{*}$ as

$$
\begin{aligned}
x_{k+1}^{* p_{k}} & =-\xi_{k}^{r_{k+1} p_{k} /\left(r_{n}+\tau_{n}\right)}\left\{(n-k+1)+\widetilde{\kappa}_{k}\left(x_{1}, \ldots, x_{k}\right)\right\} \\
& =:-\xi_{k}^{r_{k+1} p_{k} /\left(r_{n}+\tau_{n}\right)} \beta_{k}\left(x_{1}, x_{2} \ldots, x_{k}\right),
\end{aligned}
$$

then we obtain

$$
\begin{aligned}
& \dot{V}_{k}\left(x_{1}, x_{2}, \ldots, x_{k}\right) \leq-(n-k+1) \\
& \cdot\left(\xi_{1}^{2 \rho /\left(r_{n}+\tau_{n}\right)}+\cdots+\xi_{k-1}^{2 \rho /\left(r_{n}+\tau_{n}\right)}+\xi_{k}^{2 \rho /\left(r_{n}+\tau_{n}\right)}\right) \\
& \quad+\xi_{k}^{\left(2 \rho-r_{k}-\tau_{k}\right) /\left(r_{n}+\tau_{n}\right)}\left(x_{k+1}^{p_{k}}-x_{k+1}^{* p_{k}}\right)+k \delta
\end{aligned}
$$

which proves the inductive argument.

At the $n$th step, by applying the feedback control

$$
\begin{aligned}
u & =x_{n+1}=-\xi_{n} \beta_{n}\left(x_{1}, x_{2} \ldots, x_{n}\right)=-\beta_{n}(\cdot)\left(x_{n}^{\left(r_{n}+\tau_{n}\right) / r_{n}}\right. \\
& +\beta_{n-1}(\cdot)\left(x_{n-1}^{\left(r_{n}+\tau_{n}\right) / r_{n-1}}+\cdots\right. \\
& \left.\left.+\beta_{2}(\cdot)\left(x_{2}^{\left(r_{n}+\tau_{n}\right) / r_{2}}+\beta_{1}(\cdot) x^{\left(r_{n}+\tau_{n}\right) / r_{1}}\right) \ldots\right)\right)
\end{aligned}
$$

with the $C^{1}$, proper, and positive definite Lyapunov function $V_{n}\left(x_{1}, x_{2} \ldots, x_{n}\right)$ constructed via the inductive procedure, we arrive at

$$
\begin{aligned}
& \dot{V}_{n}\left(x_{1}, x_{2}, \ldots, x_{n}\right) \\
& \leq-\left(\xi_{1}^{2 \rho /\left(r_{n}+\tau_{n}\right)}+\cdots+\xi_{n-1}^{2 \rho /\left(r_{n}+\tau_{n}\right)}+\xi_{n}^{2 \rho /\left(r_{n}+\tau_{n}\right)}\right) \\
& \quad+n \delta .
\end{aligned}
$$

Recall that $V\left(x_{1}, x_{2}, \ldots, x_{n}\right)=\sum_{k=1}^{n} W_{k}\left(x_{1}, x_{2}, \ldots, x_{k}\right)$, where $W_{k}$ 's are defined in (19). Then, it follows from Lemma 6 that, for any $\sigma>0$,

$$
\begin{aligned}
V^{\sigma}\left(x_{1}, x_{2}, \ldots, x_{n}\right) \leq c \sum_{k=1}^{n} W_{k}^{\sigma}\left(x_{1}, x_{2}, \ldots, x_{k}\right), \\
\quad \forall\left(x_{1}, x_{2}, \ldots, x_{n}\right) \in R^{n},
\end{aligned}
$$

where $c:=\max \left(1, n^{\sigma-1}\right)$. 
Moreover, we have

$$
\begin{aligned}
W_{k} & \left(x_{1}, x_{2}, \ldots, x_{k}\right) \\
\leq & \left|x_{k}-x_{k}^{*}\right|\left|x_{k}^{\left(r_{n}+\tau_{n}\right) / r_{k}}-x_{k}^{*\left(r_{n}+\tau_{n}\right) / r_{k}}\right|^{\left(2 \rho-r_{k}-\tau_{k}\right) /\left(r_{n}+\tau_{n}\right)} \\
\leq & 2^{1-r_{k} /\left(r_{n}+\tau_{n}\right)}\left|x_{k}^{\left(r_{n}+\tau_{n}\right) / r_{k}}-x_{k}^{*\left(r_{n}+\tau_{n}\right) / r_{k}}\right|^{r_{k} /\left(r_{n}+\tau_{n}\right)} \\
& \cdot\left|x_{k}^{\left(r_{n}+\tau_{n}\right) / r_{k}}-x_{k}^{*\left(r_{n}+\tau_{n}\right) / r_{k}}\right|^{\left(2 \rho-r_{k}-\tau_{k}\right) /\left(r_{n}+\tau_{n}\right)} \\
\leq & 2\left|\xi_{k}\right|^{\left(2 \rho-\tau_{k}\right) /\left(r_{n}+\tau_{n}\right)}=2\left(\left|\xi_{k}\right|^{2 \rho /\left(r_{n}+\tau_{n}\right)}\right)^{\alpha},
\end{aligned}
$$

where $\alpha=\left(2 \rho-\tau_{k}\right) / 2 \rho$. Therefore,

$$
\begin{aligned}
\dot{V}_{n}\left(x_{1}, x_{2}, \ldots, x_{n}\right) \leq & -\frac{1}{2} V_{n}^{1 / \alpha}\left(x_{1}, x_{2}, \ldots, x_{n}\right)+n \delta \\
= & -\left(\frac{V_{n}\left(x_{1}, x_{2}, \ldots, x_{n}\right)}{2^{\alpha}}\right)^{1 / \alpha} \\
& +n \delta .
\end{aligned}
$$

Inequality (31) will show that the state $x(t)$ of closed-loop system (11)-(27) is well-defined on $[0,+\infty)$ and globally bounded. To prove this, first introduce the following set:

$$
\Psi=\left\{x(t) \in R^{n} \mid V_{n}(x) \geq(4 n \delta)^{\alpha}\right\},
$$

and let $x(t)$ be the trajectory of (11) with an initial state $x(0)$. If $x(t) \in \Psi$, then it follows from (32) that

$$
\dot{V}_{n}(x(t)) \leq-\frac{1}{2} V_{n}^{1 / \alpha}(x(t))+n \delta \leq-n \delta<0 .
$$

This implies that as long as $x(t) \in \Psi, V_{n}(x(t))$ is strictly decreasing with time $t$, and hence $x(t)$ must enter the complement set $R^{n}-\Psi$ in a finite time $T \geq 0$ and stay there forever. Therefore, (33) leads to

$$
\begin{gathered}
V_{n}(x(t))-V_{n}(x(0))=\int_{0}^{t} \dot{V}_{n}(x(t)) d t<0, \\
\quad t \in[0, T), \\
V_{n}(x(t))<(4 n \delta)^{\alpha}, \quad t \in[T, \infty),
\end{gathered}
$$

which shows $V_{n} \in L^{\infty}$ and so do $x_{1}$ and $W_{k}$. By $z_{1}=x_{1}+y_{r}$ and $y_{r} \in L^{\infty}$, we conclude that $x_{1} \in L^{\infty}$ as well. Noting

$$
x_{2}^{* p_{1}}=-x_{1}^{\left(r_{1}+\tau_{1}\right) / r_{1}}\left(n+\widetilde{\kappa}_{1}\left(x_{1}\right)\right)=-x_{1}^{\left(r_{1}+\tau_{1}\right) / r_{1}} \beta_{1}\left(x_{1}\right)
$$

and $\widetilde{\kappa}_{1}\left(x_{1}\right)$ is smooth function of $x_{1}$, we have $x_{2}^{* p_{1}} \in L^{\infty}$.

Since $W_{2} \in L^{\infty}$ and the inequality (21) holds, we have $\left(x_{2}-x_{2}^{*}\right) \in L^{\infty}$ and $x_{2} \in L^{\infty}$. Inductively, we can prove $x_{i} \in$ $L^{\infty}, i=3,4, \ldots, n$ and so do $x(t)$.

Thus, the solution $x(t)$ of system (11) is well-defined and globally bounded on $[0,+\infty)$.

Next, it will be shown that

$$
\left|y(t)-y_{r}(t)\right|=\left|z_{1}(t)-y_{r}(t)\right|<\varepsilon, \quad \forall t \geq T>0 .
$$

This is easily shown from (21) and (34) and by tuning the parameter $\delta$ as follows:

$$
\left|y(t)-y_{r}(t)\right|=\left|x_{1}(t)\right| \leq V_{n}(x(t)) \leq(4 n \delta)^{\alpha}<\varepsilon .
$$

Therefore, for any $\varepsilon>0$, there is globally practical output tracking such that (36) holds.

This completes the proof of Theorem 7.

\section{An Illustrative Example}

In this section, we give a simple numerical example to illustrate the correctness and effectiveness of the theoretical results by considering the following nonlinear system:

$$
\begin{aligned}
\dot{z}_{1} & =z_{2}^{7 / 3}+\lambda_{1}(t) z_{1}^{2}, \\
\dot{z}_{2} & =u+\lambda_{2}(t) z_{2}^{5 / 7} \sin z_{1}, \\
y & =z_{1}, \\
\left|\lambda_{1}(t)\right| & \leq 2, \\
\left|\lambda_{2}(t)\right| & \leq 3,
\end{aligned}
$$

where $p_{1}=7 / 3, p_{2}=1$ and $\lambda_{1}(t), \lambda_{2}(t)$ represent an unknown bounded time-varying function and parameter, respectively.

Our objective is to design a practical continuously differentiable $\left(C^{1}\right)$ output tracking controller such that the output of system (38) tracks a desired reference signal $y_{r}$, and all the states of system (38) are globally bounded.

Clearly, the system is of form (1). It is worth pointing out that although system (38) is simple, it cannot solve the global practical tracking problem using the design method presented in $[6,10]$. Choose $r_{1}=1, \tau_{1}=2 / 3$, then $r_{2}=5 / 7$, and $\tau_{2}=4 / 7$. By Lemma 4 , it is easy to obtain

$$
\begin{aligned}
\left|f_{1}(\cdot)\right| & =\left|\lambda_{1} z_{1}^{2}\right| \leq\left(1+z_{1}^{2}\right)\left|z_{1}\right|^{5 / 3}, \\
\left|f_{2}(\cdot)\right| & =\left|\lambda_{2}(t) z_{2}^{4 / 5} z_{1}^{2}\right| \\
& \leq 3\left(1+\left(1+z_{1}^{2}\right)^{2}+z_{2}^{2}\right)\left(\left|z_{1}\right|^{9 / 7}+\left|z_{2}\right|^{9 / 5}\right) .
\end{aligned}
$$

Clearly, Assumption 1 holds with $b\left(z_{1}\right)=\left(1+z_{1}^{2}\right), b\left(z_{1}, z_{2}\right)=$ $3\left(1+\left(1+z_{1}^{2}\right)^{2}+z_{2}^{2}\right)$; according to the design procedure proposed in Section 3, we can obtain a continuously differentiable $\left(C^{1}\right)$ tracking controller:

$$
\begin{aligned}
u= & -16\left(z_{2}^{9 / 5}+\beta_{1}\left(z_{1}-y_{r}\right)^{3}\right)[1 \\
& +16\left(1+\left(z_{1}-y_{r}\right)^{2}\right)^{4} \beta_{1}^{4} \\
& +\frac{4096}{\delta^{27 / 43}}\left(1+\left(z_{1}-y_{r}\right)^{2}\right)^{15} \beta_{1}^{6}+3 b_{2}\left(z_{1}-y_{2}, z_{2}\right) \\
& \left.+54 \beta_{1}^{2} b_{2}^{2}\left(z_{1}-y_{2}, z_{2}\right)+\frac{36}{\delta^{27 / 43}} b_{2}^{2}\left(z_{1}-y_{2}, z_{2}\right)\right]
\end{aligned}
$$




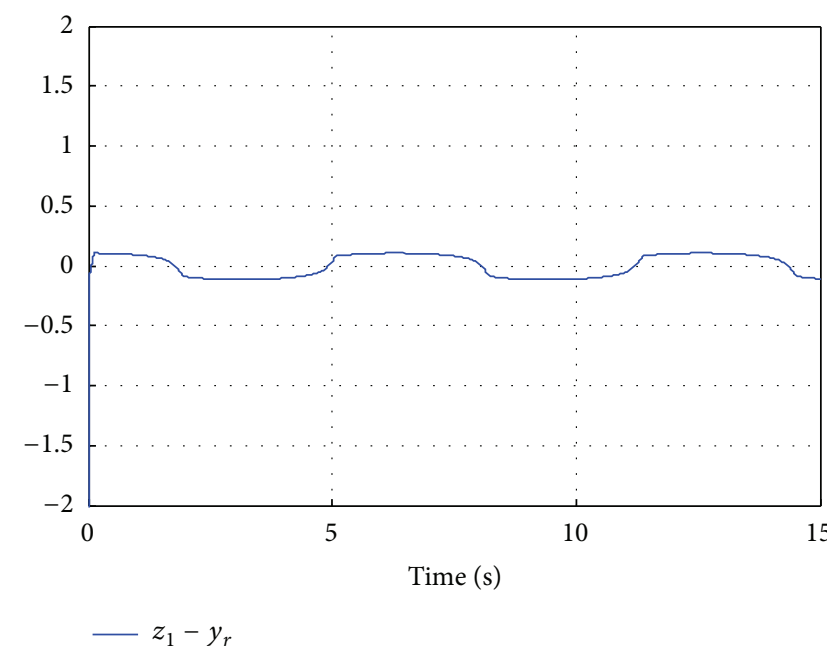

(a)

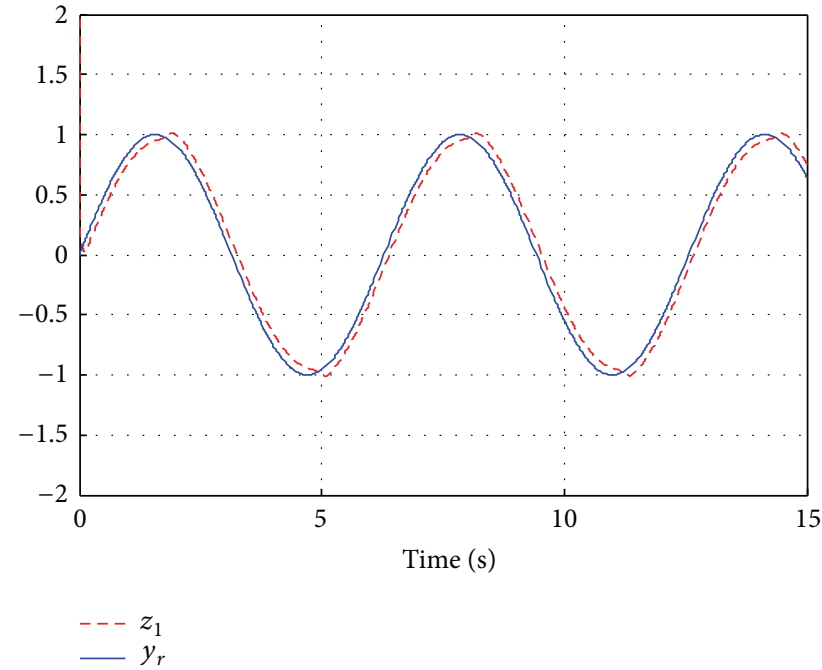

(b)

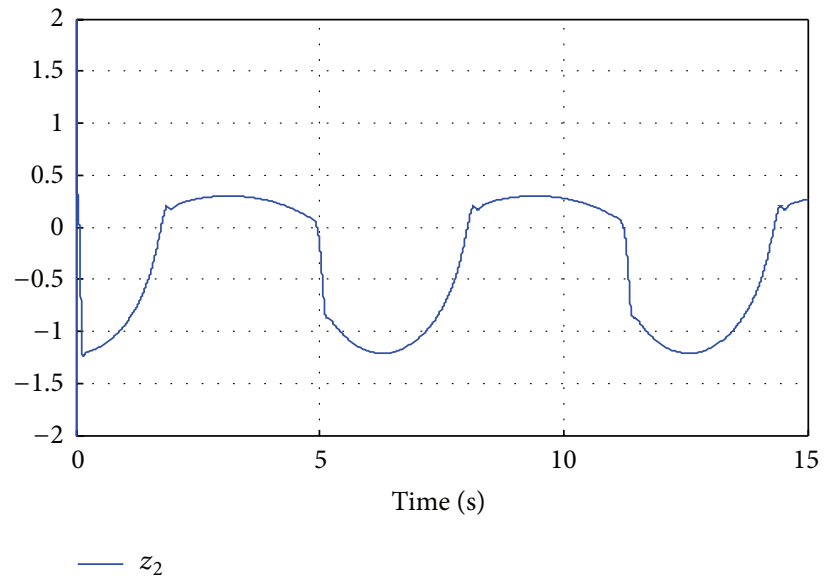

(c)

FIGURE 1: (a) Tracking error $z_{1}-y_{r}$ for $\delta=0.00225$ which result about error 0.1. (b) The trajectories of $z_{1}$ and $y_{r}$ for $\delta=0.00225$. (c) Trajectory of state $z_{2}$.

where

$$
\begin{gathered}
\beta_{1}=2+2\left(1+\left(z_{1}-y_{r}\right)^{2}\right) \\
+\frac{1}{4 \delta}\left(3+2\left(z_{1}-y_{r}\right)^{2}\right)^{2}, \\
b_{2}\left(z_{1}-y_{r}, z_{2}\right)=3\left(1+\left(1+\left(z_{1}-y_{r}\right)^{2}\right)^{2}+z_{2}^{2}\right) .
\end{gathered}
$$

In the simulation, by choosing the initial values as $z_{1}(0)=$ $4, z_{2}(0)=-3$, and $y_{r}=\sin t, \lambda_{1}(t)=2 \sin t, \lambda_{2}(t)=3$. Then, we have the following:

(i) When the parameter $\delta$ is set as $\delta=0.00225$, the tracking error obtained is about 0.1 as shown in Figures 1(a), 1(b), and 1(c). (ii) When parameter $\delta$ is increased to $\delta=0.0000225$, then the tracking error reduces to about 0.025 as shown in Figures 2(a), 2(b), and 2(c).

\section{Conclusion}

This paper has developed a systematic approach to construct a continuously differentiable $\left(C^{1}\right)$ practical output tracking controller for a class of inherently nonlinear systems, whose chained integrator part has the power of positive odd rational numbers. Such a controller guarantees that the states of the closed-loop system are globally bounded, while the tracking error can be bounded by any given positive number after a finite time. Further, a simple numerical example was performed to illustrate the effectiveness of the result obtained. 


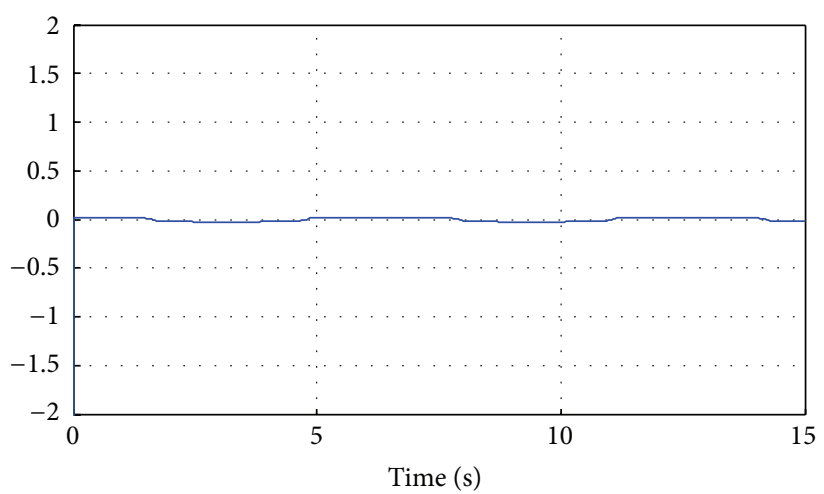

$-z_{1}-y_{r}$

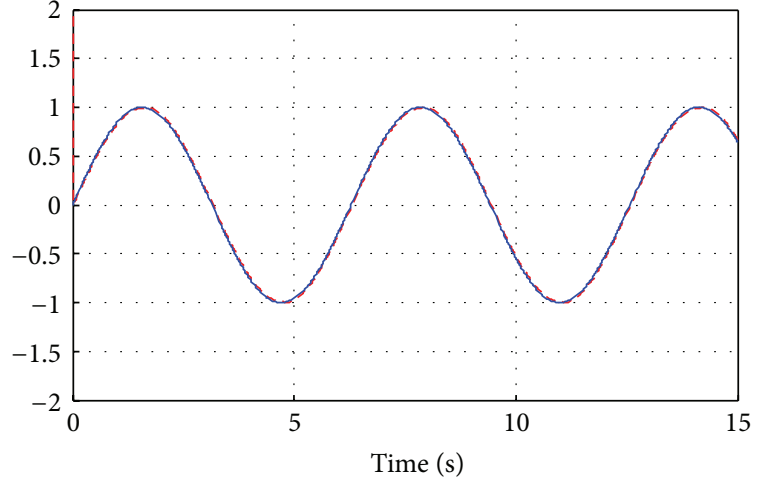

$---z_{1}$

(a)

(b)

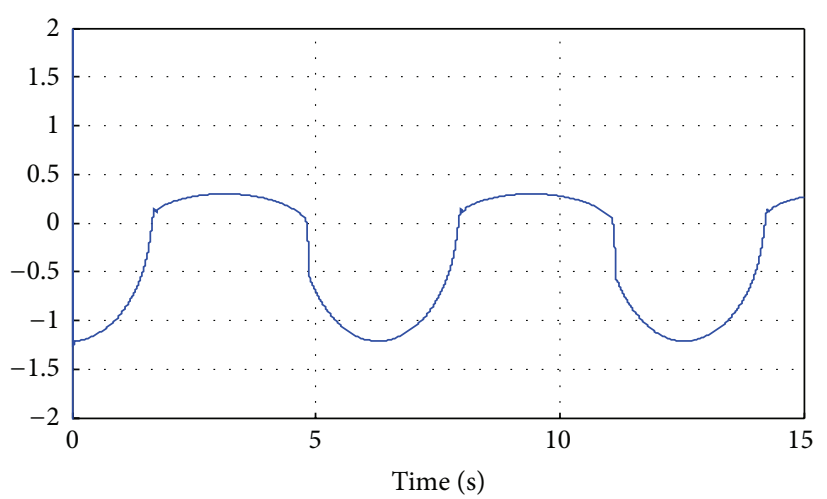

$z_{2}$

(c)

FiguRE 2: (a) Tracking error $z_{1}-y_{r}$ for $\delta=0.0000225$ which result about error 0.025. (b) The trajectories of $z_{1}$ and $y_{r}$ for $\delta=0.0000225$. (c) Trajectory of state $z_{2}$.

\section{Appendix}

Proposition A.1. There exist positive smooth functions $g_{k}\left(x_{1}, \ldots, x_{k}\right)$ such that

$$
\begin{aligned}
& \xi_{k-1}^{\left(2 \rho-r_{k-1}-\tau_{k-1}\right) /\left(r_{n}+\tau_{n}\right)}\left(x_{k}^{p_{k-1}}-x_{k}^{* p_{k-1}}\right) \\
& \quad \leq \frac{1}{3} \xi_{k-1}^{2 \rho /\left(r_{n}+\tau_{n}\right)}+g_{k}\left(x_{1}, \ldots, x_{k}\right) \xi_{k}^{2 \rho /\left(r_{n}+\tau_{n}\right)} .
\end{aligned}
$$

Proof. First, whenever $r_{k} p_{k-1} /\left(r_{n}+\tau_{n}\right) \leq 1$ it follows from Lemma 3 that

$$
\begin{aligned}
& \left(x_{k}^{p_{k-1}}-x_{k}^{* p_{k-1}}\right) \leq \mid\left(x_{k}^{\left(r_{n}+\tau_{n}\right) / r_{k}}\right)^{r_{k} p_{k-1} /\left(r_{n}+\tau_{n}\right)} \\
& -\left(x_{k}^{*\left(r_{n}+\tau_{n}\right) / r_{k}}\right)^{r_{k} p_{k-1} /\left(r_{n}+\tau_{n}\right)} \mid \\
& \leq 2^{1-r_{k} p_{k-1} /\left(r_{n}+\tau_{n}\right)} \mid x_{k}^{\left(r_{n}+\tau_{n}\right) / r_{k}} \\
& -\left.x_{k}^{*\left(r_{n}+\tau_{n}\right) / r_{k}}\right|^{r_{k} p_{k-1} /\left(r_{n}+\tau_{n}\right)}
\end{aligned}
$$

$$
\begin{aligned}
& \leq 2^{1-r_{k} p_{k-1} /\left(r_{n}+\tau_{n}\right)}\left|\xi_{k}\right|^{r_{k} p_{k-1} /\left(r_{n}+\tau_{n}\right)}=\widetilde{g}_{k}\left(x_{1}, \ldots, x_{k}\right) \\
& \cdot\left|\xi_{k}\right|^{r_{k} p_{k-1} /\left(r_{n}+\tau_{n}\right)} .
\end{aligned}
$$

By the utilization of Lemma 4 and noting that $r_{k} p_{k-1}=r_{k-1}+$ $\tau_{k-1}$, it can be seen that

$$
\begin{aligned}
& \xi_{k-1}^{\left(2 \rho-r_{k-1}-\tau_{k-1}\right) /\left(r_{n}+\tau_{n}\right)}\left(x_{k}^{p_{k-1}}-x_{k}^{* p_{k-1}}\right) \\
& \quad \leq \xi_{k-1}^{\left(2 \rho-r_{k-1}-\tau_{k-1}\right) /\left(r_{n}+\tau_{n}\right)} \tilde{g}_{k}\left(x_{1}, \ldots, x_{k}\right)\left|\xi_{k}\right|^{r_{k} p_{k-1} /\left(r_{n}+\tau_{n}\right)} \\
& \quad \leq \frac{1}{3} \xi_{k-1}^{2 \rho /\left(r_{n}+\tau_{n}\right)}+g_{k}\left(x_{1}, \ldots, x_{k}\right) \xi_{k}^{2 \rho /\left(r_{n}+\tau_{n}\right)}
\end{aligned}
$$

for a smooth function $g_{k}\left(x_{1}, \ldots, x_{k}\right)>0$. However, if $r_{k} p_{k-1} /\left(r_{n}+\tau_{n}\right) \geq 1$, by the Mean Value Theorem,

$$
\begin{gathered}
\left(x_{k}^{p_{k-1}}-x_{k}^{* p_{k-1}}\right) \leq \mid\left(x_{k}^{\left(r_{n}+\tau_{n}\right) / r_{k}}\right)^{r_{k} p_{k-1} /\left(r_{n}+\tau_{n}\right)} \\
-\left(x_{k}^{*\left(r_{n}+\tau_{n}\right) / r_{k}}\right)^{r_{k} p_{k-1} /\left(r_{n}+\tau_{n}\right)}|\leq c| x_{k}^{\left(r_{n}+\tau_{n}\right) / r_{k}}
\end{gathered}
$$




$$
\begin{aligned}
& -x_{k}^{*\left(r_{n}+\tau_{n}\right) / r_{k}} \mid\left(\left(x_{k}^{\left(r_{n}+\tau_{n}\right) / r_{k}}\right)^{r_{k} p_{k-1} /\left(r_{n}+\tau_{n}\right)-1}\right. \\
& \left.-\left(x_{k}^{*\left(r_{n}+\tau_{n}\right) / r_{k}}\right)^{r_{k} p_{k-1} /\left(r_{n}+\tau_{n}\right)-1}\right)=\tilde{g}_{k}\left(x_{1}, \ldots, x_{k}\right) \\
& \cdot\left|\xi_{k}\right|\left(\left|\xi_{k}\right|^{r_{k} p_{k-1} /\left(r_{n}+\tau_{n}\right)-1}+\left|\xi_{k-1}\right|^{r_{k} p_{k-1} /\left(r_{n}+\tau_{n}\right)-1}\right) .
\end{aligned}
$$

Finally, by Lemma 4 and again noting that $r_{k} p_{k-1}=r_{k-1}+$ $\tau_{k-1}$, it is apparent that

$$
\begin{gathered}
\xi_{k-1}^{\left(2 \rho-r_{k-1}-\tau_{k-1}\right) /\left(r_{n}+\tau_{n}\right)}\left(x_{k}^{p_{k-1}}-x_{k}^{* p_{k-1}}\right) \\
\leq \xi_{k-1}^{\left(2 \rho-r_{k-1}-\tau_{k-1}\right) /\left(r_{n}+\tau_{n}\right)} \tilde{g}_{k}\left(x_{1}, \ldots, x_{k}\right)\left|\xi_{k}\right| \\
\cdot\left(\left|\xi_{k}\right|^{r_{k} p_{k-1} /\left(r_{n}+\tau_{n}\right)-1}+\left|\xi_{k-1}\right|^{r_{k} p_{k-1} /\left(r_{n}+\tau_{n}\right)-1}\right) \\
\leq \frac{1}{3} \xi_{k-1}^{2 \rho /\left(r_{n}+\tau_{n}\right)}+g_{k}\left(x_{1}, \ldots, x_{k}\right) \xi_{k}^{2 \rho /\left(r_{n}+\tau_{n}\right)}
\end{gathered}
$$

for a smooth function $g_{k}\left(x_{1}, \ldots, x_{k}\right)>0$.

Proposition A.2. There exist positive smooth functions $h_{k}\left(x_{1}, \ldots, x_{k}\right)$ and any real number $\delta>0$ such that

$$
\begin{aligned}
& \xi_{k}^{\left(2 \rho-r_{k}-\tau_{k}\right) /\left(r_{n}+\tau_{n}\right)} f_{k}\left(x_{1}+y_{r}, x_{2}, \ldots, x_{k}\right) \\
& \leq \frac{1}{2}\left(\xi_{1}^{2 \rho /\left(r_{n}+\tau_{n}\right)}+\xi_{2}^{2 \rho /\left(r_{n}+\tau_{n}\right)}+\cdots+\xi_{k-2}^{2 \rho /\left(r_{n}+\tau_{n}\right)}\right) \\
&+\frac{1}{3} \xi_{k-1}^{2 \rho /\left(r_{n}+\tau_{n}\right)}+h_{k}\left(x_{1}, \ldots, x_{k}\right) \xi_{k}^{2 \rho /\left(r_{n}+\tau_{n}\right)} \\
&+\frac{1}{2} \delta
\end{aligned}
$$

Proof. Using Lemma 3, Assumptions 1-2 can be rewritten as (for $l=2, \ldots, k)$

$$
\begin{aligned}
& \left|f_{k}\left(x_{1}+y_{r}, x_{2}, \ldots, x_{k}\right)\right| \leq b_{k}\left(x_{1}+y_{r}, x_{2}, \ldots, x_{k}\right) \\
& \quad \cdot\left(\left|x_{1}+y_{r}\right|^{\left(r_{1}+\tau_{1}\right) / r_{1}}\right. \\
& \left.\quad+\left|x_{2}\right|^{\left(r_{2}+\tau_{2}\right) / r_{2}}+\cdots+\left|x_{k}\right|^{\left(r_{k}+\tau_{k}\right) / r_{k}}\right) \\
& \quad \leq \widetilde{b}_{k}\left(x_{1}, \ldots, x_{k}\right) \cdot\left(\left|\xi_{1}\right|^{r_{l+1} p_{l} /\left(r_{n}+\tau_{n}\right)}\right. \\
& \quad+\left|\xi_{2}-\bar{\beta}_{1} \xi_{1}\right|^{r_{l+1} p_{l} /\left(r_{n}+\tau_{n}\right)}+\cdots \\
& \left.\quad+\left|\xi_{k}-\bar{\beta}_{k-1} \xi_{k-1}\right|^{r_{l+1} p_{l} /\left(r_{n}+\tau_{n}\right)}+M^{r_{l+1} p_{l} /\left(r_{n}+\tau_{n}\right)}\right) \\
& \quad \leq \bar{b}_{k}\left(x_{1}, \ldots, x_{k}\right)\left(\left|\xi_{1}\right|^{r_{l+1} p_{l} /\left(r_{n}+\tau_{n}\right)}+\left|\xi_{2}\right|^{r_{l+1} p_{l} /\left(r_{n}+\tau_{n}\right)}\right. \\
& \left.\quad+\cdots+\left|\xi_{k}\right|^{r_{l+1} p_{l} /\left(r_{n}+\tau_{n}\right)}+M^{r_{l+1} p_{l} /\left(r_{n}+\tau_{n}\right)}\right)
\end{aligned}
$$

for smooth, positive nonzero functions $\bar{\beta}_{i}\left(x_{1}, \ldots, x_{i}\right)=$ $\beta_{i}^{\left(r_{n}+\tau_{n}\right) / r_{l+1} p_{l}}\left(x_{1}, \ldots, x_{i}\right), i=1,2, \ldots, l$, and $\bar{b}_{k}\left(x_{1}, \ldots, x_{k}\right)$.
By Lemmas 4-5 and (A.7), with $\left(2 \rho-r_{l}-\tau_{l}\right) /\left(r_{n}+\tau_{n}\right)+$ $r_{l+1} p_{l} /\left(r_{n}+\tau_{n}\right)=2 \rho /\left(r_{n}+\tau_{n}\right)$,

$$
\begin{gathered}
\xi_{l}^{\left(2 \rho-r_{l}-\tau_{l}\right) /\left(r_{n}+\tau_{n}\right)} f_{l}\left(x_{1}+y_{r}, x_{2}, \ldots, x_{l}\right) \\
\quad \leq\left|\xi_{l}\right|^{\left(2 \rho-r_{l}-\tau_{l}\right) /\left(r_{n}+\tau_{n}\right)} \bar{b}_{k}\left(x_{1}, \ldots, x_{k}\right)
\end{gathered}
$$$$
\cdot\left(\left|\xi_{1}\right|^{r_{l+1} p_{l} /\left(r_{n}+\tau_{n}\right)}+\left|\xi_{2}\right|^{r_{l+1} p_{l} /\left(r_{n}+\tau_{n}\right)}+\cdots\right.
$$$$
\left.+\left|\xi_{k}\right|^{r_{l+1} p_{l} /\left(r_{n}+\tau_{n}\right)}+M^{r_{l+1} p_{l} /\left(r_{n}+\tau_{n}\right)}\right) \leq \frac{1}{2}\left(\xi_{1}^{2 \rho /\left(r_{n}+\tau_{n}\right)}\right.
$$$$
\left.+\xi_{2}^{2 \rho /\left(r_{n}+\tau_{n}\right)}+\cdots+\xi_{k-2}^{2 \rho /\left(r_{n}+\tau_{n}\right)}\right)+\frac{1}{3} \xi_{k-1}^{2 \rho /\left(r_{n}+\tau_{n}\right)}
$$$$
+\left(\widehat{b}_{k}\left(x_{1}, \ldots, x_{k}\right)+\widehat{B}_{k}\left(x_{1}, \ldots, x_{k}\right)\right) \xi_{k}^{2 \rho /\left(r_{n}+\tau_{n}\right)}
$$$$
+\frac{1}{2} \delta
$$

for a smooth function $h_{k}\left(x_{1}, \ldots, x_{k}\right) \geq \widehat{b}_{k}\left(x_{1}, \ldots, x_{k}\right)+$ $\widehat{B}_{k}\left(x_{1}, \ldots, x_{k}\right)>0$ and any real number $\delta>0$.

Proposition A.3. There exist positive smooth functions $l_{k}\left(x_{1}, \ldots, x_{k}\right)$ and any real number $\delta>0$ such that

$$
\begin{aligned}
\left|\sum_{i=1}^{k-1} \frac{\partial W_{k}}{\partial x_{i}} \dot{x}_{i}\right| & \\
\leq & \frac{1}{2}\left(\xi_{1}^{2 \rho /\left(r_{n}+\tau_{n}\right)}+\xi_{2}^{2 \rho /\left(r_{n}+\tau_{n}\right)}+\cdots+\xi_{k-2}^{2 \rho /\left(r_{n}+\tau_{n}\right)}\right) \\
& +\frac{1}{3} \xi_{k-1}^{2 \rho /\left(r_{n}+\tau_{n}\right)}+l_{k}\left(x_{1}, \ldots, x_{k}\right) \xi_{k}^{2 \rho /\left(r_{n}+\tau_{n}\right)}+\frac{1}{2} \delta .
\end{aligned}
$$

Proof. Consider

$$
\begin{aligned}
& \left|\frac{\partial W_{k}}{\partial x_{l}} \dot{x}_{l}\right| \\
& \quad \leq c\left|x-x_{k}^{*}\right|\left|\xi_{k}\right|^{\left(2 \rho-r_{k}-\tau_{k}\right) /\left(r_{n}+\tau_{n}\right)-1}\left|\frac{\partial x_{k}^{*\left(r_{k}+\tau_{k}\right) / r_{k}}}{\partial x_{l}} \dot{x}_{l}\right| \\
& \quad \leq c\left|\xi_{k}\right|^{\left(2 \rho-\tau_{k}-r_{n}-\tau_{n}\right) /\left(r_{n}+\tau_{n}\right)}\left|\frac{\partial x_{k}^{*\left(r_{k}+\tau_{k}\right) / r_{k}}}{\partial x_{l}} \dot{x}_{l}\right|
\end{aligned}
$$

where the last inequality is from Lemma 3 with $p=\left(r_{n}+\right.$ $\left.\tau_{n}\right) / r_{k} \geq 1$. 
By definition of $x_{k}^{*}$ and Lemma 4 , we have

$$
\begin{aligned}
& \frac{\partial x_{k}^{*\left(r_{n}+\tau_{n}\right) / r_{k}}}{\partial x_{l}}=\frac{\partial\left(\bar{\beta}_{k-1}\left(x_{1}, \ldots, x_{k-1}\right) \xi_{k-1}\right)}{\partial x_{l}} \\
& =c_{k-1}\left(x_{1}, \ldots, x_{k-1}\right) \frac{\partial\left(x_{l}^{\left.\left(r_{n}+\tau_{n}\right) / r_{l}\right)}\right.}{\partial x_{l}} \\
& =c_{k-1}\left(x_{1}, \ldots, x_{k-1}\right) x_{l}^{\left(r_{n}+\tau_{n}-r_{l}\right) / r_{l}} \\
& =c_{k-1}\left(x_{1}, \ldots, x_{k-1}\right) \\
& \cdot\left(\xi_{l}+x_{l}^{*\left(r_{n}+\tau_{n}\right) / r_{l}}\right)^{\left(r_{n}+\tau_{n}-r_{l}\right) /\left(r_{n}+\tau_{n}\right)} \\
& =c_{k-1}\left(x_{1}, \ldots, x_{k-1}\right)\left(\xi_{l}+\bar{\beta}_{l-1} \xi_{l-1}\right)^{\left(r_{n}+\tau_{n}-r_{l}\right) /\left(r_{n}+\tau_{n}\right)} \\
& \leq \widetilde{c}_{k-1}\left(x_{1}, \ldots, x_{k-1}\right) \sum_{i=l-1}^{l}\left|\xi_{i}\right|^{\left(r_{n}+\tau_{n}-r_{l}\right) /\left(r_{n}+\tau_{n}\right)} .
\end{aligned}
$$

Thus, it follows from (A.7) giving $r_{l+1} p_{l}=r_{l}+\tau_{l}$ that

$$
\begin{aligned}
\left|\frac{\partial x_{k}^{*}\left(r_{n}+\tau_{n}\right) / r_{k}}{\partial x_{l}}\right| \leq \widetilde{c}_{k-1}\left(x_{1}, \ldots, x_{k-1}\right) \\
\quad \cdot \sum_{i=l-1}^{l}\left|\xi_{i}\right|^{\left(r_{n}+\tau_{n}-r_{l}\right) /\left(r_{n}+\tau_{n}\right)} \cdot\left(\left|x_{l+1}\right|^{p_{l}}\right. \\
\left.+\sum_{j=1}^{l}\left|\xi_{j}\right|^{r_{l+1} p_{l} /\left(r_{n}+\tau_{n}\right)}+M^{r_{l+1} p_{l} /\left(r_{n}+\tau_{n}\right)}\right) \\
\quad \leq \widetilde{c}_{k-1}\left(x_{1}, \ldots, x_{k-1}\right) \sum_{i=l-1}^{l}\left|\xi_{i}\right|^{\left(r_{n}+\tau_{n}-r_{l}\right) /\left(r_{n}+\tau_{n}\right)} \\
+\left(\left|\xi_{l+1}+x_{l+1}^{*\left(r_{n}+\tau_{n}\right) / r_{l+1}}\right|^{r_{l+1} p_{l} /\left(r_{n}+\tau_{n}\right)}\right. \\
\left.+\sum_{j=1}^{l}\left|\xi_{j}\right|^{r_{l+1} p_{l} /\left(r_{n}+\tau_{n}\right)}+M^{r_{l+1} p_{l} /\left(r_{n}+\tau_{n}\right)}\right) \\
\quad+\bar{c}_{k-1}\left(x_{1}, \ldots, x_{k-1}\right) \sum_{i=l-1}^{l}\left|\xi_{i}\right|^{\left(r_{n}+\tau_{n}-r_{l}\right) /\left(r_{n}+\tau_{n}\right)} \\
\left.+\sum_{j=1}^{l}\left|\xi_{j}\right|^{r_{l+1} p_{l} /\left(r_{n}+\tau_{n}\right)}+M^{r_{l+1} p_{l} /\left(r_{n}+\tau_{n}\right)}\right)
\end{aligned}
$$

Further, it follows from Lemma 4 and the above fact that

$$
\begin{aligned}
& \left|\frac{\partial x_{k}^{*\left(r_{n}+\tau_{n}\right) / r_{k}}}{\partial x_{l}}\right| \leq \widehat{c}_{k-1}\left(x_{1}, \ldots, x_{k-1}\right) \\
& \cdot\left(\sum_{i=1}^{l+1}\left|\xi_{i}\right|^{\left(r_{n}+\tau_{n}-r_{l}+r_{l+1} p_{l}\right) /\left(r_{n}+\tau_{n}\right)}\right. \\
& \left.+M^{\left(r_{n}+\tau_{n}-r_{l}+r_{l+1} p_{l}\right) /\left(r_{n}+\tau_{n}\right)}\right)=\widehat{c}_{k-1}\left(x_{1}, \ldots, x_{k-1}\right) \\
& \quad\left(\sum_{i=1}^{l+1}\left|\xi_{i}\right|^{\left(r_{n}+\tau_{n}+\tau_{l}\right) /\left(r_{n}+\tau_{n}\right)}+M^{\left(r_{n}+\tau_{n}+\tau_{l}\right) /\left(r_{n}+\tau_{n}\right)}\right) .
\end{aligned}
$$

Under the realization that $\tau_{l} \geq \tau_{k}, \forall l=1, \ldots, k$, the following inequalities can be obtained from Lemmas $4-5$ :

$$
\begin{aligned}
& \left|\frac{\partial W_{k}}{\partial x_{i}} \dot{x}_{i}\right| \leq \overline{\mathcal{c}}_{k-1}\left(x_{1}, \ldots, x_{k-1}\right)\left|\xi_{k}\right|^{\left(2 \rho-\tau_{k}-r_{n}-\tau_{n}\right) /\left(r_{n}+\tau_{n}\right)} \\
& \quad \cdot\left|\frac{\partial x_{k}^{*\left(r_{k}+\tau_{k}\right) / r_{k}}}{\partial x_{l}} \dot{x}_{l}\right| \leq \overline{\mathcal{c}}_{k-1}\left(x_{1}, \ldots, x_{k-1}\right) \\
& \cdot\left|\xi_{k}\right|^{\left(2 \rho-\tau_{k}-r_{n}-\tau_{n}\right) /\left(r_{n}+\tau_{n}\right)} \\
& \quad \cdot\left(\sum_{i=1}^{l+1}\left|\xi_{i}\right|^{\left(r_{n}+\tau_{n}+\tau_{l}\right) /\left(r_{n}+\tau_{n}\right)}+M^{\left(r_{n}+\tau_{n}+\tau_{l}\right) /\left(r_{n}+\tau_{n}\right)}\right) \\
& \quad \leq \frac{1}{2}\left(\xi_{1}^{2 \rho /\left(r_{n}+\tau_{n}\right)}+\xi_{2}^{2 \rho /\left(r_{n}+\tau_{n}\right)}+\cdots+\xi_{k-2}^{2 \rho /\left(r_{n}+\tau_{n}\right)}\right) \\
& \quad+\frac{1}{3} \xi_{k-1}^{2 \rho /\left(r_{n}+\tau_{n}\right)}+l_{k}\left(x_{1}, \ldots, x_{k}\right) \xi_{k}^{2 \rho /\left(r_{n}+\tau_{n}\right)}+\frac{1}{2} \delta
\end{aligned}
$$

for smooth, strictly positive functions $l_{k}\left(x_{1}, \ldots, x_{k}\right)$ and any real number $\delta>0$.

\section{Conflict of Interests}

The authors declare that there is no conflict of interests regarding the publication of this paper.

\section{Acknowledgment}

This work was supported in part by JSPS KAKENHI Grant no. 26420428.

\section{References}

[1] A. Isidori and C. I. Byrnes, "Output regulation of nonlinear systems," IEEE Transactions on Automatic Control, vol. 35, no. 2, pp. 131-140, 1990.

[2] C. I. Byrnes, F. D. Priscoli, and A. Isidori, Output Regulation of Uncertain Nonlinear Systems, Birkhäauser, Boston, Mass, USA, 1997.

[3] Q. Gong and C. Qian, "Global practical output regulation of a class of nonlinear systems by output feedback," in Proceedings of the 44th IEEE Conference on Decision and Control, and the 
European Control Conference (CDC-ECC '05), pp. 7278-7283, IEEE, Seville, Spain, December 2005.

[4] X. Yan and Y. Liu, "Global practical tracking by output-feedback for nonlinear systems with unknown growth rate," Science China. Information Sciences, vol. 54, no. 10, pp. 2079-2090, 2011.

[5] X. Yan and X. Song, "Global practical tracking by output feedback for nonlinear systems with unknown growth rate and time delay," The Scientific World Journal, vol. 2014, Article ID 713081, 7 pages, 2014.

[6] C. Qian and W. Lin, "Practical output tracking of nonlinear systems with uncontrollable unstable linearization," IEEE Transactions on Automatic Control, vol. 47, no. 1, pp. 21-36, 2002.

[7] W. Lin and R. Pongvuthithum, "Adaptive output tracking of inherently nonlinear systems with nonlinear parameterization," IEEE Transactions on Automatic Control, vol. 48, no. 10, pp. 1737-1749, 2003.

[8] K. Alimhan and H. Inaba, "Robust practical output tracking by dynamic output feedback for uncertain non-linear systems with unstabilisable and undetectable linearisation," International Journal of Modelling, Identification and Control, vol. 5, no. 1, pp. 1-13, 2008.

[9] K. Alimhan and H. Inaba, "Robust practical output tracking by output compensator for a class of uncertain inherently nonlinear systems," International Journal of Modelling, Identification and Control, vol. 4, no. 4, pp. 304-314, 2008.

[10] K. Alimhan and N. Otsuka, "A note on practically output tracking control of nonlinear systems that may not be linearizable at the origin," Communications in Computer and Information Science, vol. 256, pp. 17-25, 2011.

[11] B. Yang and W. Lin, "Robust output feedback stabilization of uncertain nonlinear systems with uncontrollable and unobservable linearization," IEEE Transactions on Automatic Control, vol. 50, no. 5, pp. 619-630, 2005.

[12] J. Polendo and C. Qian, "A generalized homogeneous domination approach for global stabilization of inherently nonlinear systems via output feedback," International Journal of Robust and Nonlinear Control, vol. 17, no. 7, pp. 605-629, 2007.

[13] J. Back, S. G. Cheong, H. Shim, and J. H. Seo, "Nonsmooth feedback stabilizer for strict-feedback nonlinear systems that may not be linearizable at the origin," Systems and Control Letters, vol. 56, no. 11-12, pp. 742-752, 2007.

[14] J. Polendo and C. Qian, "A universal method for robust stabilization of nonlinear systems: unification and extension of smooth and non-smooth approaches," in Proceedings of the American Control Conference, pp. 4285-4290, June 2006.

[15] K. Alimhan and N. Otsuka, "A new result on practically output tracking control of nonlinear systems that may not be linearizable at the origin," in Proceedings of the 8th IEEE International Symposium on Instrumentation and Control Technology (ISICT '12), pp. 235-239, July 2012.

[16] S. P. Bhat and D. S. Bernstein, "Continuous finite-time stabilization of the translational and rotational double integrators," IEEE Transactions on Automatic Control, vol. 43, no. 5, pp. 678-682, 1998.

[17] V. T. Haimo, "Finite time controllers," SIAM Journal on Control and Optimization, vol. 24, no. 4, pp. 760-770, 1986.

[18] J. Polendo, Global synthesis of highly nonlinear dynamic systems with limited and uncertain information [Ph.D. thesis], University of Texas at San Antonio, 2006.

[19] C. Qian and W. Lin, "Recursive observer design, homogeneous approximation, and nonsmooth output feedback stabilization of nonlinear systems,' IEEE Transactions on Automatic Control, vol. 51, no. 9, pp. 1457-1471, 2006.

[20] C. Qian and W. Lin, "Non-Lipschitz continuous stabilizers for nonlinear systems with uncontrollable unstable linearization," Systems \& Control Letters, vol. 42, no. 3, pp. 185-200, 2001.

[21] C. Qian and W. Lin, "A continuous feedback approach to global strong stabilization of nonlinear systems," IEEE Transactions on Automatic Control, vol. 46, no. 7, pp. 1061-1079, 2001. 


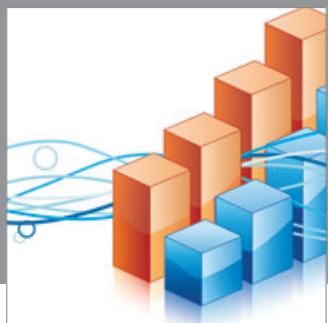

Advances in

Operations Research

mansans

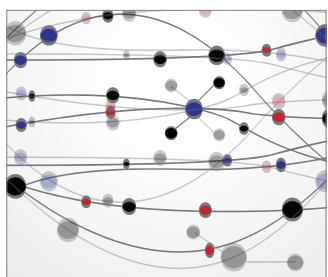

The Scientific World Journal
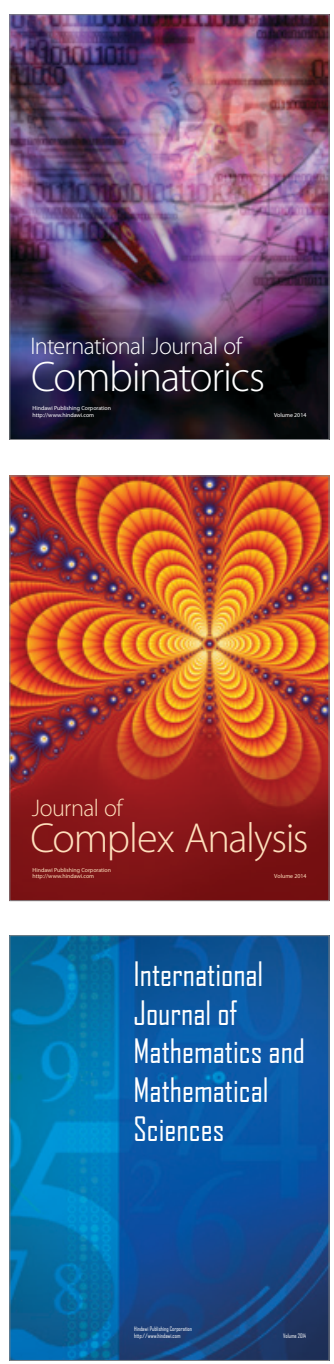
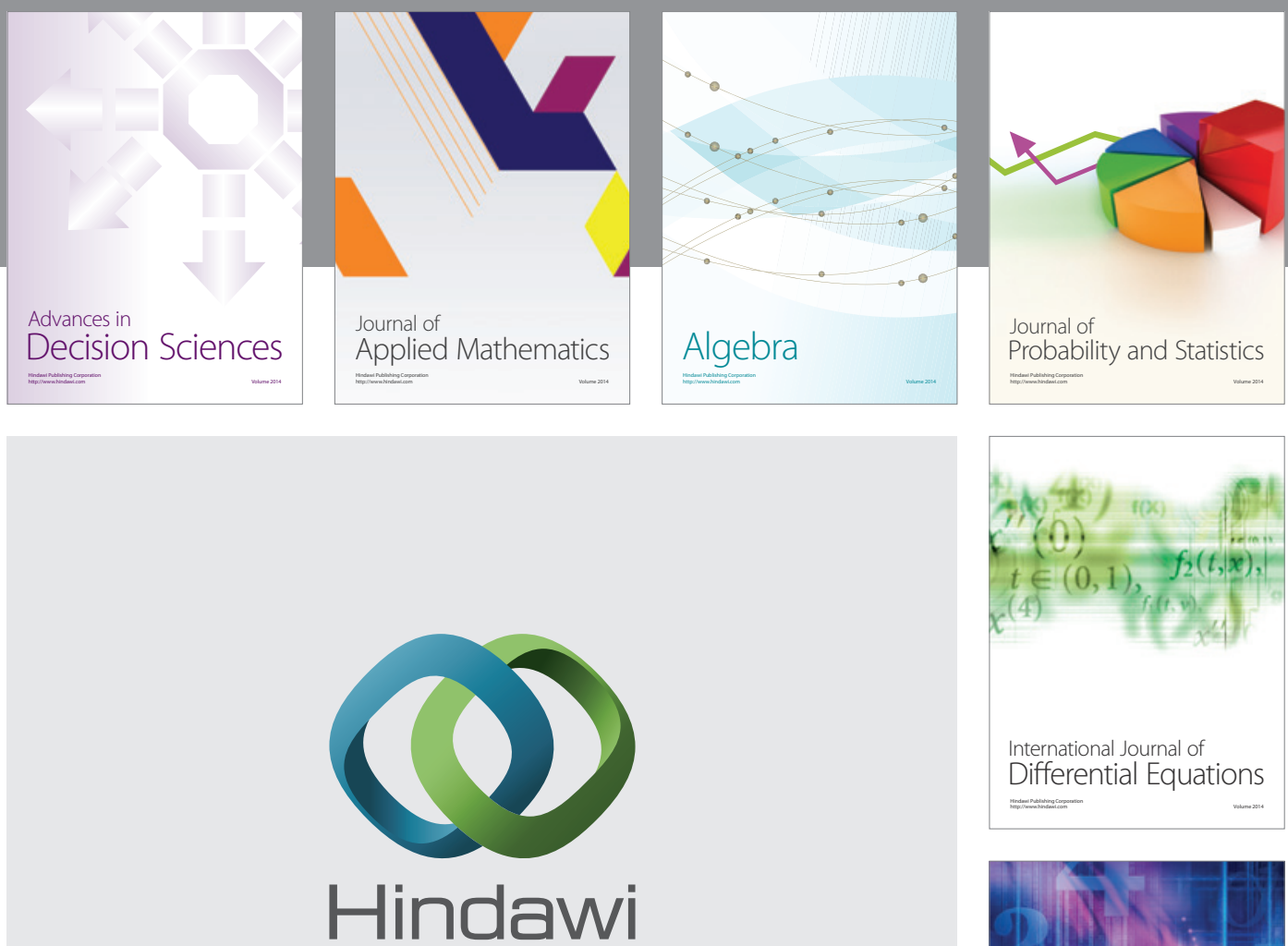

Submit your manuscripts at http://www.hindawi.com
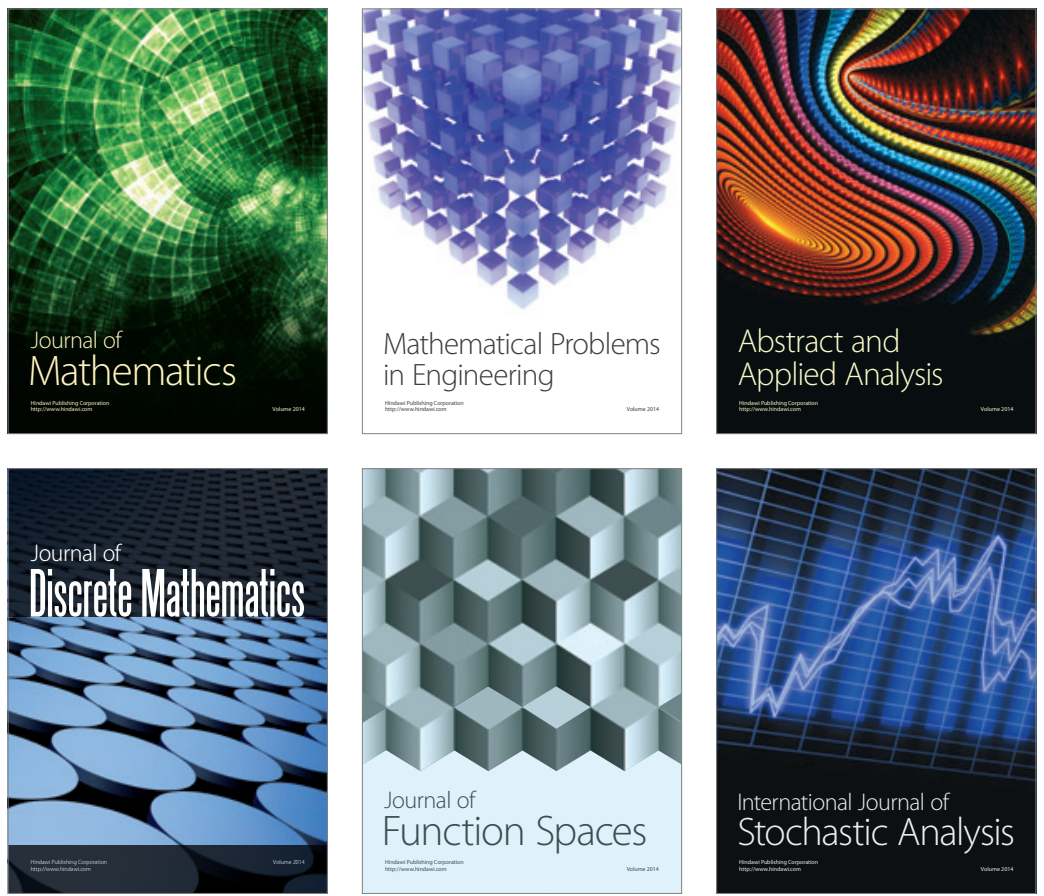

Journal of

Function Spaces

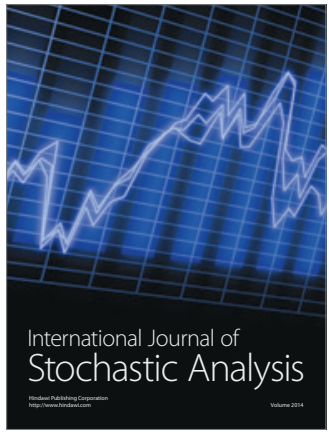

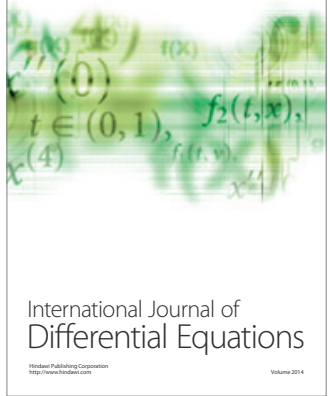
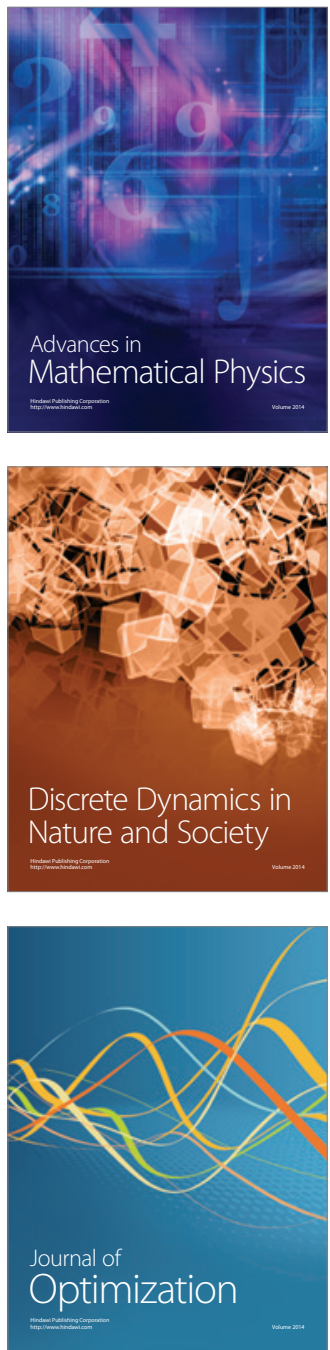